\title{
A Design for a Novel Open, Intelligent and Integrated CNC System Based on ISO 10303-238 and PMAC
}

\author{
Yu ZHANG, Qifeng ZENG, Guodong MU, Yafei YANG, Yutao YAN, Wanli SONG, Yadong GONG
}

\begin{abstract}
The combination of the high-level data model called ISO 10303-238 with the open programmable multi-axis controller (PMAC) presents a vision for the open, intelligent and integrated computer numerical control (CNC) systems whose demands have been growing with the rapid development of modern manufacturing. Evolved from design philosophy, this paper proposes a novel open, intelligent and integrated CNC system based on ISO 10303-238 and PMAC. In the system, ISO 10303-238 is chosen as the numerical control (NC) data in order to make the CNC system interoperable. And the open master-slave hardware structure on the basis of industrial process computer (IPC) + PMAC with double central processing units (CPUs) is designed in order to make the CNC system flexible. Also, the open and modular software structure is designed in order to make the CNC system intelligent. In addition, the development of the prototype system is given. At the end, it has been verified by case study that the proposed CNC system is feasible and effective.
\end{abstract}

Keywords: intelligent CNC system; ISO 10303-238; PMAC

\section{INTRODUCTION}

As one of the advanced manufacturing technologies, $\mathrm{CNC}$ has been extensively applied to various machine tools on account of the efficient and precise machining and easy operation. And, it becomes a core element in a modern manufacturing system.

Up to now, most commercial CNC systems are based upon G\&M code which is dated back to 1950s. G\&M code is a low-level data model which primarily points out the tool-path [1]. Because it only provides limited information to $\mathrm{CNC}$, it makes $\mathrm{CNC}$ nothing but an actuating mechanism. Moreover, because G\&M code is machine-dependent, it makes CNC non-portable and noninteroperable. Another issue is that it supports unidirectional information flow from design to manufacturing, which makes the information at the workshop level not be fed back to the designer and breaks the chain among $\mathrm{CAD} / \mathrm{CAPP} / \mathrm{CAM} / \mathrm{CNC}$. What is more, these constraints impede the development of the open, intelligent and integrated $\mathrm{CNC}$ systems which are essential for contemporary manufacturing system. To solve the problems arising from G\&M code, International Standardization Organization (ISO) is developing a highlevel NC data model called standard for the exchange of product data-compliant numerical control (STEP-NC) [2, 3]. Other than G\&M code, this high-level data model uses the object-oriented 'Workingsteps' which corresponds to advanced machining features and related process parameters to describe machining processes. That is to say, implementing STEP-NC can realize the transition from the task-level data to the method-level data and keep all the machining information such as geometry, feature, tolerance, machine tool and cutters information. So far, much significant work about STEP-NC-based CNC systems has been done by a lot of scholars and institutions. However, most research about STEP-NCbased CNC systems [4-14] is compliant with the ISO 14649 which makes the open, intelligence and integration of the CNC systems inadequacy. Moreover, the open and real-time of these systems is restricted because traditional $\mathrm{NC}$ controller or soft $\mathrm{CNC}$ is used. Hence, there is a requirement for high-level data model to be developed and utilized that can enhance the open, intelligence and integration of the CNC systems. Furthermore, there is also a need to make a balance between the open and real time of the CNC. Thus, a novel open, intelligent and integrated CNC system based on ISO 10303-238 and PMAC is proposed in this paper.

The remainder of this paper is organized as follows. Section 2 presents three crucial considerations for designing the open, intelligent and integrated CNC. Derived from design considerations, a data model to be used for the system is given and the framework of the novel open, intelligent and integrated $\mathrm{CNC}$ system based on ISO 10303-238 and PMAC is proposed and studied in Section 3. On the basis of the presented framework, implementation details and implementation status are given in Section 4. Section 5 demonstrates a case study. In Section 6, conclusions and future works are given.

\section{DESIGN PHILOSOPHY}

Designing the open, intelligent and integrated CNC, three crucial aspects involving with $\mathrm{NC}$ data, $\mathrm{CNC}$ functions and $\mathrm{CNC}$ implementations need to be considered. The requirements for every aspect are summed up as follows.

\subsection{Requirements for NC Data}

The NC data is concerned with data interface scheme between CAD, CAPP, CAM and $\mathrm{CNC}$, and data manipulation within $\mathrm{CNC}$. Therefore, the requirements for NC data are given as follows.

Providing full machining information for $\mathrm{CNC}$ : The full machining information including machining features information, process planning information, cutting tool information and workpiece properties and so forth enables the $\mathrm{CNC}$ to carry out a true adaptable, optimized and smart control. Therefore, the complete information's requirement must exist for realizing an intelligent and autonomous CNC. 


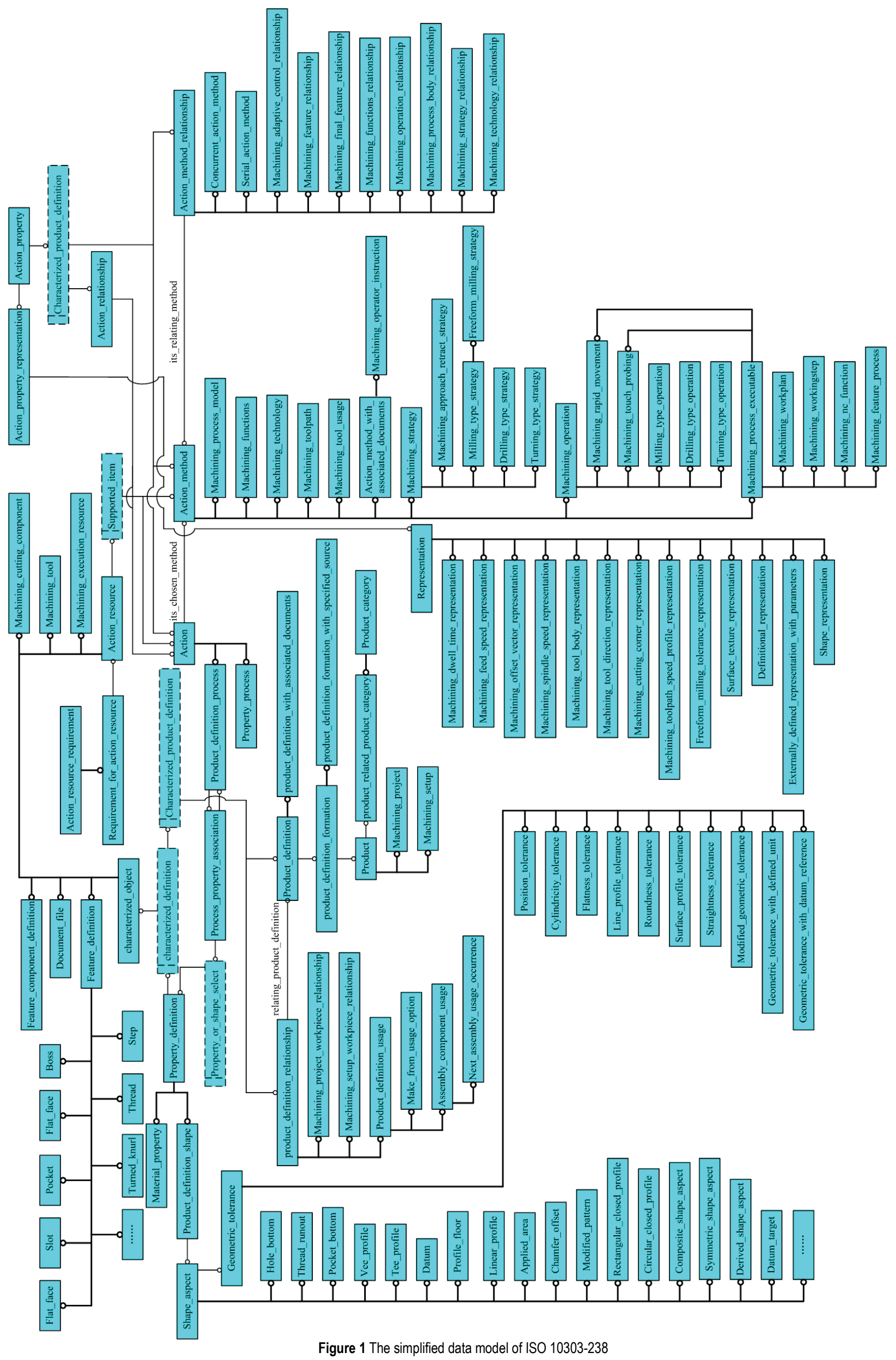


(2) Supporting two-way information flow among CAD/CAPP/CAM/CNC: Frequently, an NC program modified in the workshop differs from that generated by the $\mathrm{CAD} / \mathrm{CAPP} / \mathrm{CAM}$ system. For feeding back the modification in the workshop to upstream systems, an NC data for supporting two-way information flow between $\mathrm{CAD}, \mathrm{CAPP}, \mathrm{CAM}$ and $\mathrm{CNC}$ is required.

(3) Providing data portability between CNCs: Conventional NC programs are proprietary and machine tool-dependent, which makes an obstacle for cooperation among manufacturing partners. To resolve this situation, the data portability between CNCs is required so that $\mathrm{NC}$ programs can be applied to any $\mathrm{CNC}$ machine tools capable of performing the machining tasks.

(4) Supporting data expansion for new machining processes: Data expansion will be made for the integration of other advanced manufacturing approaches such as $3 \mathrm{D}$ printing and high precision machining that are presently very promising, whereas whose implementations are still restricted because of the defects from the innovation of $\mathrm{NC}$ technology such as incomplete cutting theories, and slow and unintelligent $\mathrm{NC}$ programming.

(5) Supporting data sharing through the Internet: The data sharing through the Internet is an essential condition for achieving a series of networked manufacturing technologies such as remote monitoring and distributed manufacturing. To enable the manufacturing technologies, there is a need that $\mathrm{NC}$ data can be easily accessed via the Internet.

\subsection{Requirements for CNC Functions}

The requirements of $\mathrm{CNC}$ functions are associated to activities of $\mathrm{CNC}$ to control machine tools, by which the part can be machined accurately during the machining process. Considering the activities intelligent and integrated, the following aspects are taken into account.

(1) Integratability: As a powerful platform, the $\mathrm{CNC}$ can be integrated with CAD/CAPP/CAM.

(2) Autonomy: The CNC is capable of creating NC programs, planning machining sequences, generating tool paths and executing generated tool paths, etc. with little human intervention.

(3) Intelligence: The $\mathrm{CNC}$ is capable of optimizing machining plans, checking collisions between workpiece, fixture, cutting tool and machine tool, monitoring machining statuses such as vibration, acceleration, cutting power and feed-rate, adapting variable machining conditions, inspecting in-process and post-process workpiece, dealing with unexpected events such as abnormal tool breakage and tool wear, and acquiring machining knowledge, etc.

(4) Manageability: All the resources concerned with the process of the machining should be controlled and managed.

\subsection{Requirements for CNC Implementations}

The requirements of $\mathrm{CNC}$ implementations contain the requirements of software implementations and the requirements of hardware implementations.

The requirements of software implementations concern the structure and topology of software modules and the implementation methodologies. The prime concerns here are the modularity, modifiability, portability and friendliness of software modules and easyto-use implementation tools.

(1) Modularity: For operating in a plug and play fashion, the software modules should be developed on the basis of modular structure.

(2) Modifiability: To perfect the functions, the CNC should enable the users to have access to the software modules and make any modifications.

(3) Portability: Because computer environments utilized by the users are not always identical, the developed software modules need to be able to support all the heterogeneous platforms.

(4) Friendliness: The software modules are demanded to easily interact with the users by GUI, diagnostic message and visual validation.

(5) Easy-to-use implementation tools: For the CNC is a very complex system, a modelling tool which is easy to be used is demanded to ease the design burden. Also, there is a need for a good software platform and programming method.

In order to guarantee the flexibility of the hardware in the whole system, a flexible hardware should be established.

\section{DESIGN OF THE SYSTEM 3.1 Applying ISO 10303-238 into CNC}

The data model is vital to realize the open, intelligent and integrated CNC. Other than G\&M code, STEP-NC developed by ISO is a high-level object-oriented data model for CNC, which uses the concept of 'Workingstep'. It is feature-cantered and contains all the machining information such as the machining features information, cutting tools information and machining operations information and so on. Nowadays, STEP-NC is being developed by two different ISO subcommittees considering two different focuses. ISO 14649 (ARM version of STEP-NC) is developed by ISO TC 184/SC1, which is more possible to be used where CAM systems can obtain exact information from the workshop, while ISO 10303-238 (AIM version of STEP-NC) is done by ISO TC $184 / \mathrm{SC} 4$, which is more suitable to be used in an integrated design and manufacturing environment. Considering the requirements of NC data, the ISO 10303238 is chosen as the data model of the CNC. Fig. 1 shows its simplified data model.

\subsection{The Framework for the Open and Modular Hardware Based on IPC and PMAC}

In terms of above considerations, the open and modular "IPC + PMAC"-based hardware framework is come up with, as shown in the Fig. 2. In the framework, looked as the upper control unit, the IPC is a PC-based computing platform which has advanced features such as expansion, reliability and compatibility for industrial applications. It is in charge of dealing with the non-realtime tasks such as process planning and tool-path planning. Correspondingly, considered as the lower control unit, the PMAC developed by the Delta Tau is a computer which has abilities of independent operation and motion control as well as advanced features such as 
reliability, compatibility and flexibility. It is in charge of dealing with real-time tasks such as position control, speed control and interpolation. The PCI bus and DPRAM are used for the communication between IPC (master computer) and PMAC (slave computer). Hence, the framework of the hardware supports the development and extension of the $\mathrm{CNC}$, and provides the two-level openness for the CNC.

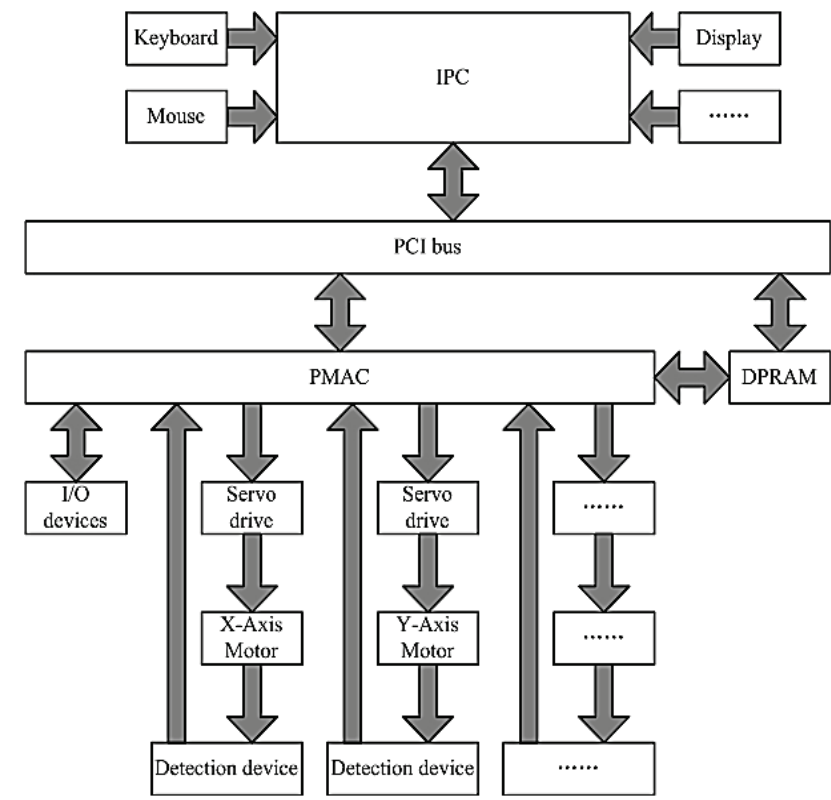

Figure 2 The framework for the open and modular hardware based on IPC and PMAC

\subsection{The Framework for the Open and Modular Software Based on ISO 10303-238}

Based upon the above analysis, the open and modular software framework shown in the Fig. 3 is put forward in this section, which consists of man machine interaction (MMI) module, communication module, database modules, non-real-time modules and real-time modules.

\subsubsection{Module}

MMI module offers friendly interactive functions between the operator and the $\mathrm{CNC}$ system. Through it, the $\mathrm{NC}$ program, machining parameters and operation commands, etc. can be input to the CNC system by the operator. At the same time, the $\mathrm{CNC}$ system also can display the present machining status, speed information and position information, etc. to the operator through it. That is to say, it serves as a console of the CNC system that the operator uses: (1) to create and load the STEP-NC program, (2) to set up the machine tools, (3) to perform the operation of machine tools, and (4) to monitor the operation status for efficient machining.

\subsubsection{Communication Module}

The communication module is in charge of finish the data transmission between IPC and PMAC.

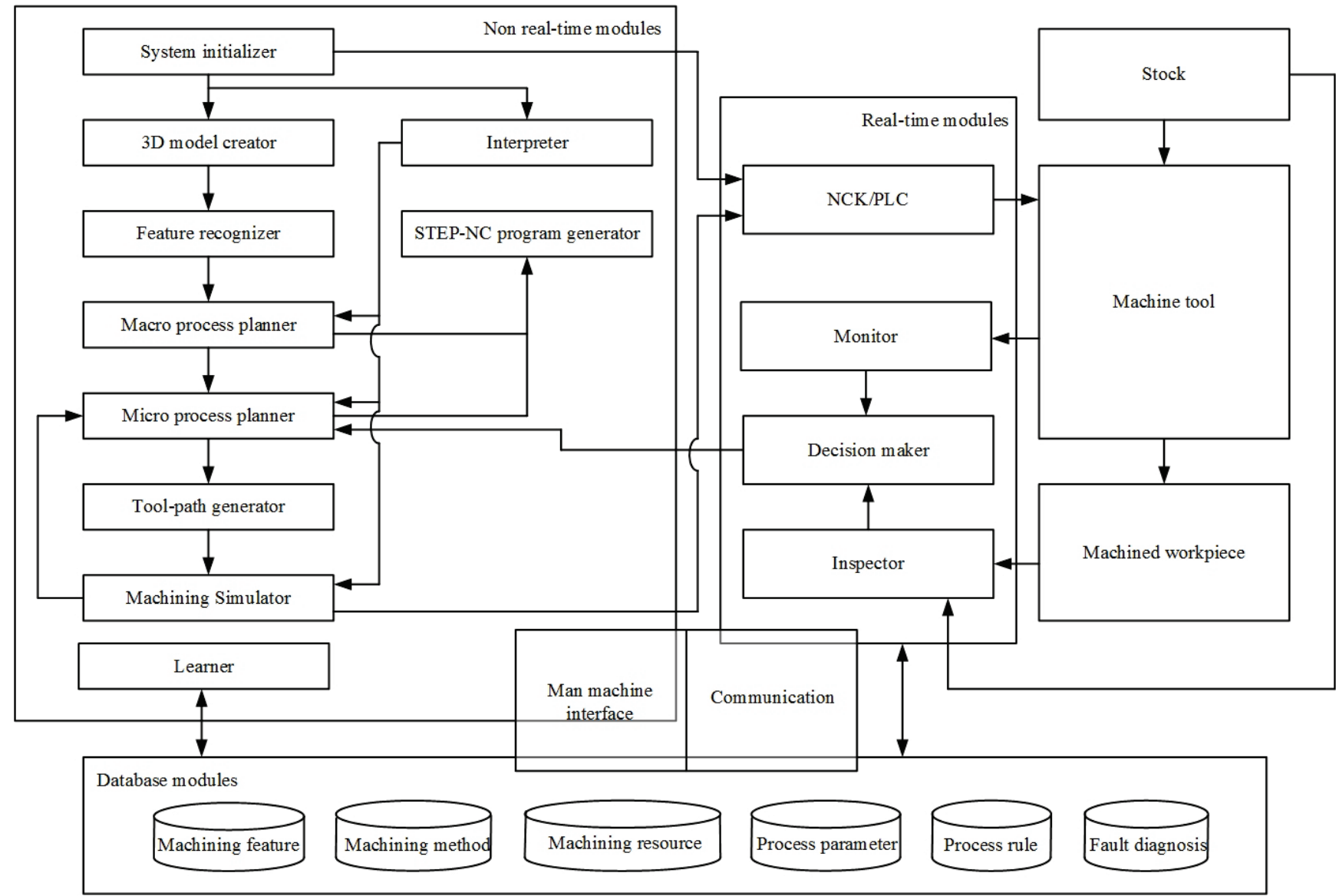

Figure 3 The framework for the open and modular software based on ISO 10303-238 


\subsubsection{Database Modules}

Taking the machining feature as the core based on the relationships of the entities, database modules are the data repositories generated and used by non-real-time modules and real-time modules. It contains machining feature database, machining method database, machining resource database, process parameter database, process rule database and fault diagnosis database.

(1) Machining feature database. This stores the machining feature information such as planar face, pocket, slot, round hole, boss, outer round and knurl conforming to STEP-NC standard. Stored data is the core data for the process planning.

(2) Machining method database. This store all the machining operation information involved with the specific machining environment, which covers operation methods such as end milling, plane milling, bottom and side milling, tapping, machining strategies such as freedom strategy, drilling type strategy and two5D milling strategy, and approach and retract strategies such as bidirectional strategy and contour parallel milling strategy. Stored data is used for the process planning.

(3) Machining resource database. This stores the machine tool data such as general properties, capabilities and machine configuration, the cutter data such as the angle of the cutter, the radius of the edge, the tooth number, the coolant and the material, and the fixture and sensor data. It can be modified by the operator.

(4) Process parameter database. This stores the process parameter data such as feedrate and spindle speed which exists in the process manual or is standardized and relatively fixed in the engineering. It can be updated by the learner which analyses and acquires the machining knowledge based on neural network expert system.

(5) Process rule database. This stores the process rule data involved with the process decision-making, including the principles for selecting the machining allowance, machining method, process parameter, machining resource and process sequence, etc. It can be updated by the learner.

(6) Fault diagnosis database: This stores the fault diagnosis knowledge. It can be updated by the learner.

\subsubsection{Non-Real-Time Modules}

Non-real-time modules run in the IPC are responsible for finishing non-real-time tasks which cover system initialization, three-dimensional modelling, feature recognition, process planning, STEP-NC program generation, STEP-NC program interpretation, tool-path generation and machining simulation. Main non-real-time modules are described as follows.

(1) System initializer. The system initializer is in charge of initializing the PMAC, e.g. loading PComm32 dynamic link library, setting the communication mode and communication address between IPC, PMAC and DPRAM, and giving the initial values for the CNC system.

(2) 3D model creator. The 3D model creator takes charge of creating the 3D model of the workpiece and generating the corresponding STEP AP-203 file which includes the geometrical and topological information of the workpiece to be used by the feature recognizer.

(3) Feature recognizer. The function of feature recognizer is to extract the geometrical and topological information of the workpiece from the STEP AP-203 file, further recognize machining features in terms of the machining feature schema defined in the STEP AP-224 and generate the STEP AP-224 file containing machining feature information to be applied to the process planning.

(4) Macro process planner. The role of macro process planner is to plan the macro process planning through selecting suitable machining operations for machining features. The output of the macro process planner is the STEP AP-240 file. The generative macro process planning is independent of machine tools and can be applied to the generation of the generic STEP-NC program.

(5) Micro process planner. The role of micro process planner is to enrich the macro process planning by pointing out the stock, setups, machining sequences, machining parameters and available machining resources like fixtures, cutters and machine tools. Therefore, the generated micro process planning can meet the machining requirements at the workshop level. Its workflow is described as follows. First, every feature and its corresponding machining operation are integrated into a workingstep. After these workingsteps are ordered and combined, some auxiliary workingsteps and operations such as rapid approach are inserted, which make all the workingsteps continuous. Then, machining parameters from available machining resources are added to the micro process planning. Therefore, a specific process planning is created. The output of the micro process planner is the STEP AP-240 file. The generative micro process planning is dependent on machine tools and can be applied to the generation of the specific STEP-NC program.

(6) STEP-NC program generator. STEP-NC program generator is in charge of generating part programs in ISO 10303-21 or ISO 10303-28 format. The generated program describes advanced machining features and associated machining parameters in an object-oriented manner with the use of the concept of 'Workingsteps' When the machining information involved with the macro process planning are mapped into the file in ISO 1030321 or ISO 10303-28 format, a generic STEP-NC program can be generated. When the machining information concerned with the micro process planning is mapped into the file in ISO $10303-21$ or ISO 10303-28 format, a specific STEP-NC program can be generated and directly used in the workshop.

(7) Interpreter. The interpreter is to take the STEP$\mathrm{NC}$ program as an input and translate it according to the defined schema.

(8) Tool-path generator. The tool-path generator takes charge of generating a complete tool-paths including approach paths, machining paths, retract paths and connection paths between workingsteps based upon the information of process planning. The generated tool paths are accessed by NCK/PLC with the capability of NURBS interpolation.

(9) Machining simulator. The machining simulator aims at verifying the specific machining data generated by the above modules before it is applied to the actual machining. It has two major functions. On the one side, it graphically shows the cutting process of the workpiece 
and examines any possible errors such as workpiece undercut and collision between machine tool, cutter and workpiece. On the other side, based on mathematical models with physical factors and geometric information in the machining process, it predicts the cutting forces, feed-rate, vibration and surface roughness, etc.

(10) Learner. It analyses and acquires the machining knowledge based on neural network expert system and incorporates (stores) it into the databases.

\subsubsection{Real-Time Modules}

Real-time modules run in PMAC are responsible for finishing real-time tasks which cover data transformation, interpolation, monitoring, decision making as well as inspection. Main real-time modules for which PMAC provides basic functions are described as follows.

(1) NCK/PLC. NCK/ PLC is in charge of transforming the STEP-NC data into a set of position, motion and function commands, and performing them to activate the servo mechanism, tool change mechanism as well as workpiece loading/unloading mechanism and so forth. Also, NCK/ PLC can implement NURBS interpolation by which high speed and high accuracy machining is able to be achieved with reduced data.

(2) Monitor. The monitor is to continuously monitor the complete machining process and provide the machining status by capturing information from sensors. The results are sent to the decision maker.

(3) Decision maker. Its function is to make decisions based on the information gained from the monitor and inspector, and arranges the machining tasks. One of the crucial decisions is to designate the priorities between the planned task and the newly called task.

(4) Inspector. The inspector is able to carry out automatically inspections during the pre-process, inprocess and post-process. In any case, the inspection is executed by on-machine measurement (OMM). Also, through comparing the inspected data with the designed data, it is able to find out geometric errors between the designed part and the machined part.

\section{DEVELOPMENT OF A PROTOTYPE CALLED OICS}

On the basis of the above proposed framework, a Microsoft Windows-based prototype system called OICS was developed using the object-oriented modelling techniques. The main programming language is Java, and Java 3D is used for visualization. In addition, PComm32 [15] is used to develop real-time modules, which is a dynamic link library developed by the Delta Tau. Because it provides more than 400 functions, users can efficiently and easily develop real-time modules. In the following, technical and implementary details are given for the following non-real-time modules considering the importance of their development.

\subsection{Interpreter for ISO 10303-238 Program}

On the basis of the structure shown in Fig. 4, the interpreter for ISO 10303-238 program was developed. The main workflow of the interpreter is as follows. First of all, the interpreter reads/compiles the physical file. Then, instances of classes in accordance with the entities defined in the schema is created and the member values of instances is set through referring to the attributes of the entities. Finally, the interpreted data are saved to their respective databases. Fig. 5 displays the tree structure of the data from an interpreted program.

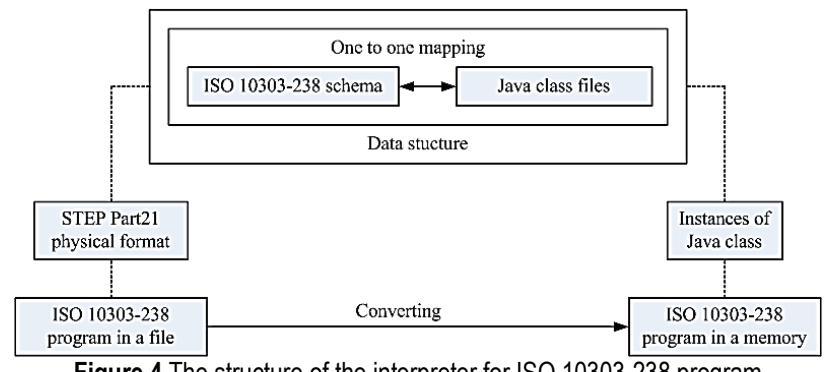

Figure 4 The structure of the interpreter for ISO 10303-238 program

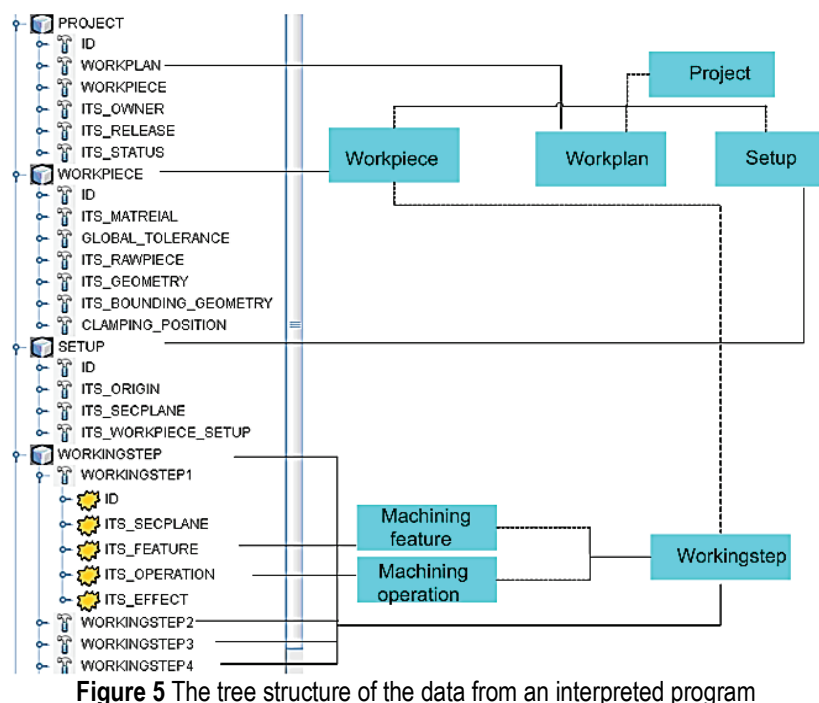

\subsection{Feature Recognizer}

Graph pattern matching is a distinct B-Rep-based feature recognition approach [16]. In this paper, the approach was applied to recognize the features defined in ISO 10303-238. The workflow of the feature recognizer is: (1) extracting the geometrical and topological information from the STEP AP203 file, (2) searching the boundary information, (3) matching the feature boundary model, and (4) generating and combining the feature. Fig. 6 shows an implementation of the feature recognizer. In the implementation, all three features of the part, i.e., planar_face, closed_pocket and round_hole are successfully recognized.

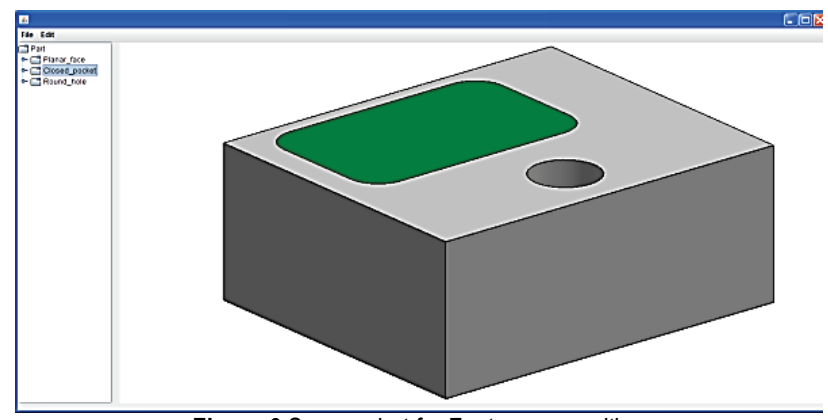

Figure 6 Screen shot for Feature recognition 


\subsection{Process Planner}

The process planner was developed based on BP neural network. The main workflow of the process planner is as follows. First of all, machining operations are designated to machining features. Then, the workingsteps are set up by combining machining features with corresponding machining operations. Subsequently, the workingsteps are classified into several groups according to the setup and are ordered. Finally, machining parameters are added to the workingsteps according to available manufacturing resources. Fig. 7 displays the screen shots of an implementation from the process planner.

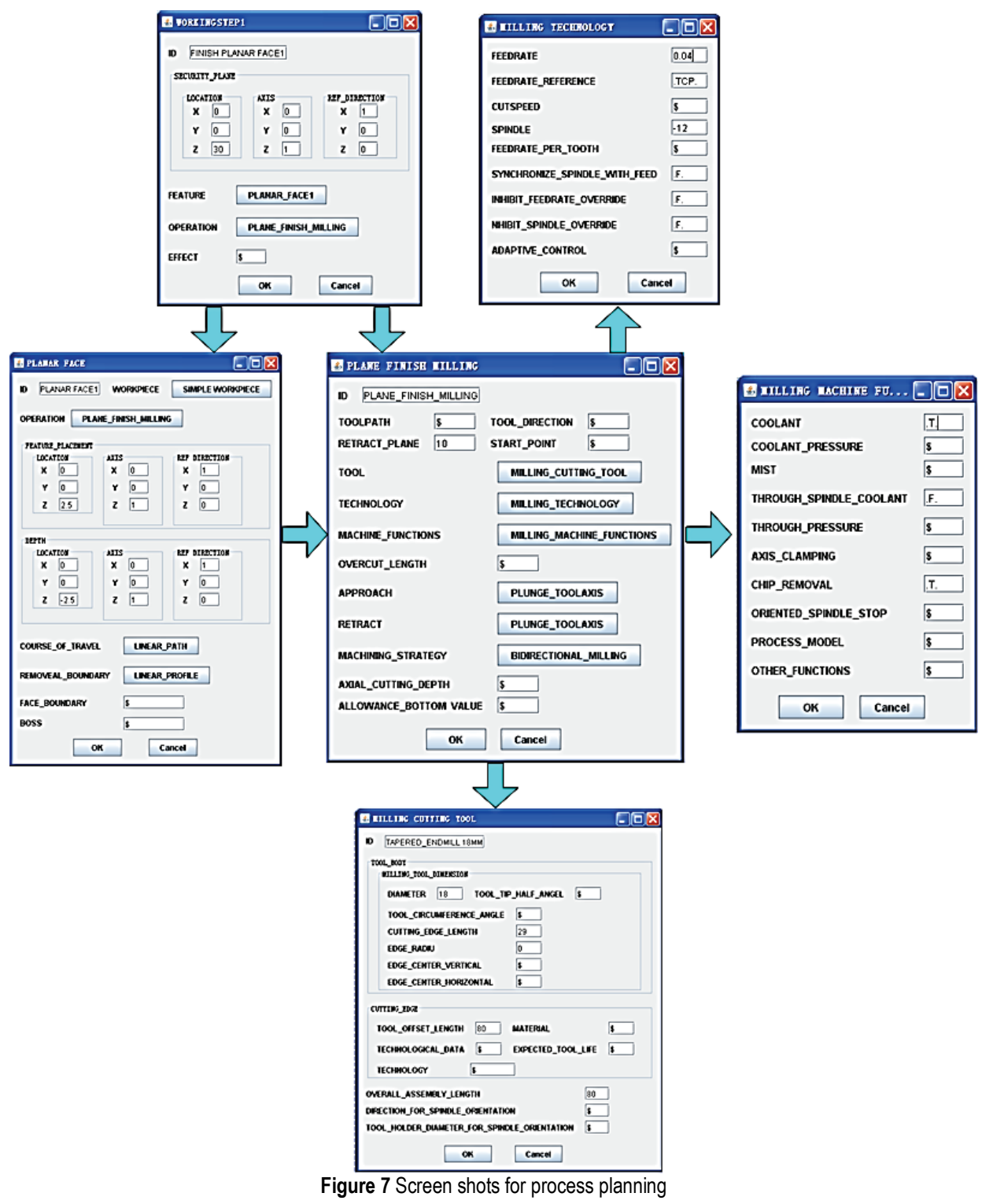

\subsection{Tool-Path Generator}

Tool-path information in the STEP-NC program is optional, and it is not given in most cases. Therefore, toolpath generation capability needs to be added to the CNC. According to the STEP-NC data model, the tool-path is composed of four segments: approach, machining, retract, and departure. Tool-path generator generates the tool path for each segment based on the machining strategy such as 'plunge_zigzag' tool approach and 'bidirectional' machining path and so on specified by the ISO 10303-238 program. Fig. 8 shows the tool-path generated by toolpath generator.

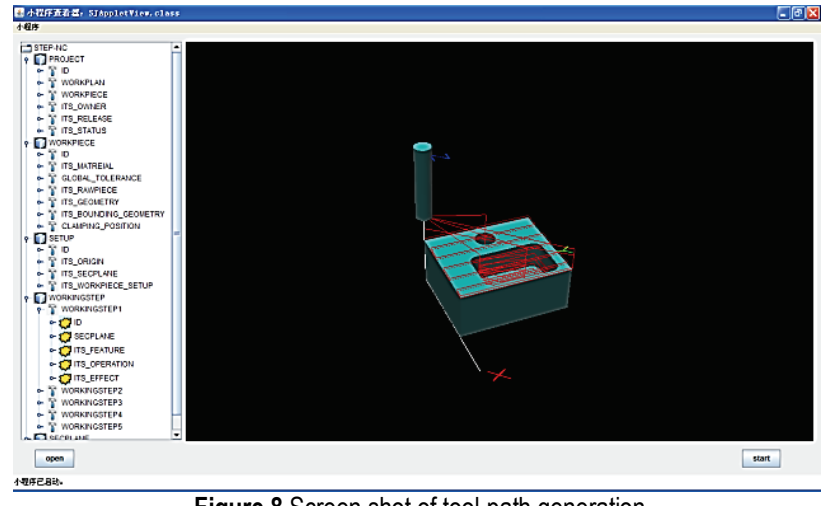

Figure 8 Screen shot of tool-path generation 


\subsection{ISO 10303-238 Program Generator}

ISO $10303-238$ program is generated by mapping the geometry information, feature information and process planning information into the program according to ISO $10303-21$ or ISO $10303-28$. The generated program is composed of two sections: HEADER section and DATA

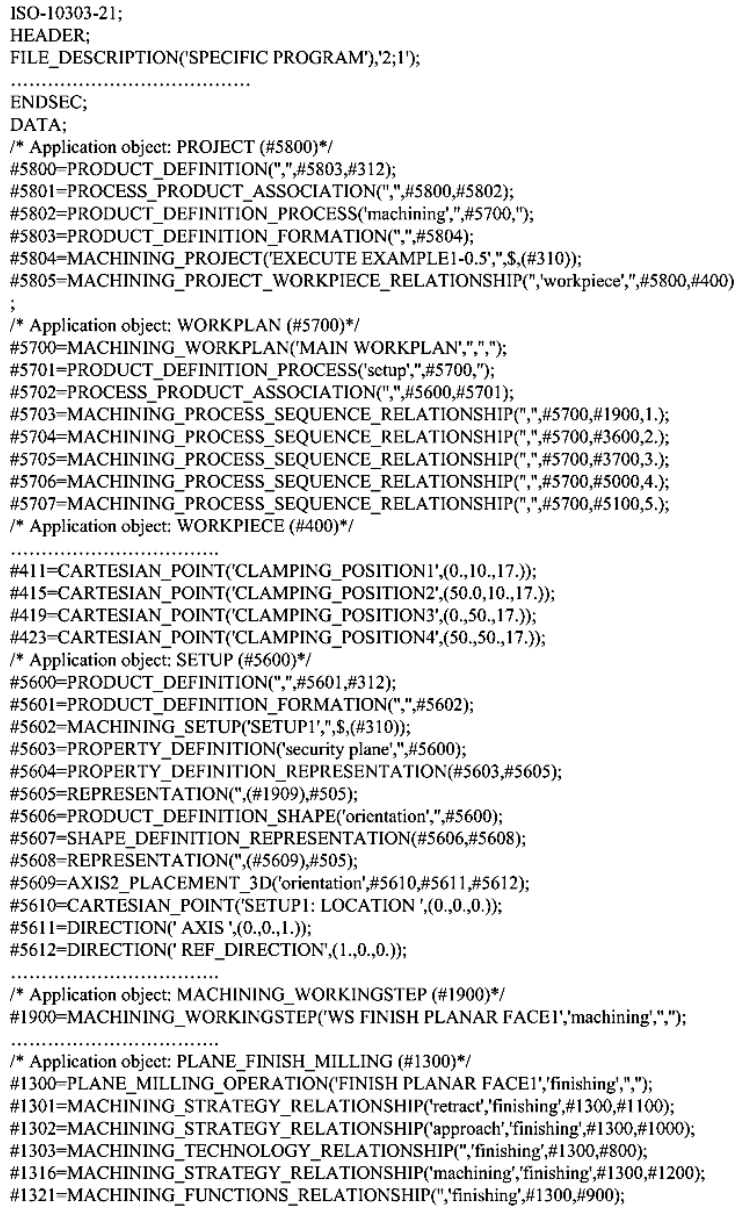

Figure 9 Generated ISO 10303-238 program

\section{CASE STUDY}

Fig. 10 shows the model of the part used in this case study, created in UG NX 4.0. After the model was exported as a STEP AP-203 file and imported into OICS, the feature recognition, process planning, tool-path planning and $\mathrm{NC}$ program generation were done as summarized from Fig. 5 to Fig. 9. After the generative tool path was performed by NCK/PLC, the part was machined. Fig. 11 shows the machined result, verifying the feasibility and effectiveness of the proposed OICS.

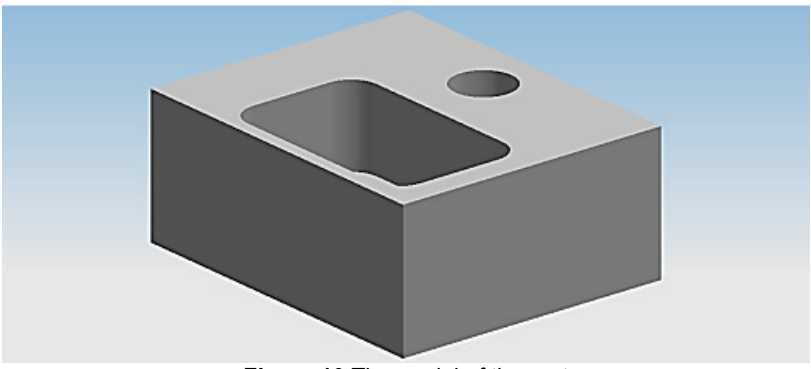

Figure 10 The model of the part section. HEADER section is involved in file name, file description and file mode. DATA section contains all the machining information and associated process parameters, which is major part of the program. Fig. 9 shows the generated ISO 10303-238 program.
\#600=MACH object: ENDMILL $(\# 600)^{* /}$

$\# 606=$

\#607=ACTION_RESOURCE_RELATIONSHIP(",", \#600, \#500);

$\# 608=A C T I O N \_R E S O U R C E \_R E L A T I O N S H I P(",,, \# 600, \# 500)$,

\#609=RESOURCE_PROPERTY('tool body',",\#600);

610=RESOURCE_PROPERTY_REPRESENTATION(",",\#609,\#700),

611 =ACTION_RESOURCE_TYPE('milling cutting tool');

\#700=MACHINING_TOOL_BODY_REPRESENTATION('endmill',( $\$ 702, \# 701, \# 703, \# 704$

$705, \# 706, \# 707), \# 505)$;

701=DESCRIPTIVE_REPRESENTATION_ITEM('coolant through tool',

' 'D03-MEASURE_REPRESENTATION_ITEM( hand of cut',right'); COUNT_MEASURE(4.), \#434);

*Application object: MILLING_TECHNOLOGY $(\# 800)^{* /}$

800=MACHINING_TECHNOLOGY (",'milling',",",

$801=$ ACTION PROPERTY('synchronize spindle with feed','milling', \#800);

$\# 802=$ ACTION PROPERTY REPRESENTATION ("','milling',\#801, \#803);

803=REPRESËETATION(","\#804),\#505);

804=DESCRIPTIVE REPRESENTATION ITEM(",'not synchronized")

P05=ACTION_PROPERTY('fodmot',

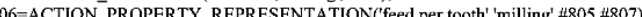
1807=MACHINING FEFD SPEFD REPRESENTATION('foed per tooth'(4808), \#808=MEASURE_REPRESENTATION_ITEM('feed per tooth',S,\#833);

\#809=ACTION_PROPERTY('spindle','milling',\#800);

$810=$ ACTION PROPERTY REPRESENTATION('rotational speed','milling', \#809, \#811);

811=MACHINING SPINDLE_SPEED REPRESENTATION('spindle speed',(\#812),\#505);

*Application object: MILLING MACHINE FUNCTIONS (\#900)*/

$900=$ MACHINING FUNCTIONS(",'milling',",");

901 =ACTION PROPERTY(chip removal ', 'milling', $\# 900) ;$

902=ACTION PROPERTY REPRESENTATION ("'milling', \#901, \#903);

903=REPRESENTATION('constant',(\#904),\#505);

904=DESCRIPTIVE REPRESENTATION ITEM('constant','chip removal on');

$905=$ ACTION PROPERTY('coolant','milling', \#900);

(960)=ACTION_PROPERTY_REPRESENTATION(",'milling',\#905,\#907);

(constant', $(\# 908), \# 505)$;

Application object: PLUNGE RAMP ( $\# 1000)^{*} /$

作,

* Application object: PLUNGE RAMP (

\#1100=MACHINING_APPROACH_RETRACT_STRATEGY(",'plunge ramp',",");

* Application object: BIDIRECTIONAL (\#1200)*/

\#1200=MILLING_TYPE_STRATEGY(",bidirectional',",")

ENDSEC

END-ISO-10303-21; 
compatible with the STEP standard supports the open, intelligent and integrated $\mathrm{CNC}$ system at the data level. The adoption of the IPC and PMAC with openness, scalability and powerful performances guarantees it at the hardware level. The utilization of the concept of modularity groups machining processes and machining activities and sustains it at the software level. The CNC framework presented in this paper has been tested and verified by a prototype system. Although it is a prototype, the core technology required for the CNC system is fully incorporated in it. Currently, full implementation for the $\mathrm{CNC}$ system on the basis of the proposed scheme is under development. More details will be given in a future paper. In addition, research works will be extended to 4 or 5 -axis $\mathrm{CNC}$ system in the future.

\section{Acknowledgements}

This project is supported by National Natural Science Foundation of China (Grant No. 51205054), China Postdoctoral Science Foundation Funded Project (2017M611245) and the Fundamental Research Funds for the Central Universities (Grant No. N160304009).

\section{REFERENCES}

[1] Zhang, Y., Xu, X. \& Liu, Y. X. (2011). Numerical control machining simulation: a comprehensive survey. International Journal of Computer Integrated Manufacturing, 24(7), 593-609. https://doi.org/10.1080/0951192X.2011.566283

[2] ISO 14649-1. (2003). Industrial automation systems and integration - physical device control - data model for computerized numerical controllers -- part 1: overview and fundamental principles, International Organization for Standardization.

[3] ISO 10303-238. (2007). Industrial automation systems and integration - product data representation and exchange -part 238: application protocol: application interpreted model for computerized numerical controllers, International Organization for Standardization.

[4] Weck, M. \& Wolf, J. (2003). ISO 14649 provides information for sophisticated and flexible numerically controlled production. Production Engineering, 10(2), 4146.

[5] Heusinger, S., Rosso, Jr. R. S. U., Klemm, P., Newman, S. T. \& Rahimifrad, S. (2006). Integrating the CAx process chain for STEP-compliant NC manufacturing of asymmetric parts. International Journal of Computer Integrated Manufacturing, 19(6), 533-545. https://doi.org/10.1080/09511920600622098

[6] Suh, S. H. \& Cheon, S. U. (2002). A framework for an intelligent $\mathrm{CNC}$ and data model. The International Journal of Advanced Manufacturing Technology, 19(10), 727-735. https://doi.org/10.1007/s001700200083

[7] Suh, S. H., Cho, J. H. \& Hong, H. D. (2002). On the architecture of intelligent STEP-compliant CNC. International Journal of Computer Integrated Manufacturing, 15(2), 168-177. https://doi.org/10.1080/09511920110056541

[8] Suh, S. H., Chung, D. H., Lee, B. E., Cho, J. H., Cheon, S. U., Hong, H. D. \& Lee, H. S. (2002). Developing an integrated STEP-compliant CNC prototype. Journal of Manufacturing Systems, 21(5), 350-362. https://doi.org/10.1016/S0278-6125(02)80034-6

[9] Suh, S. H., Chung, D. H., Lee, B. E., Shin, S., Choi, I. \& Kim, K. M. (2006). STEP-compliant CNC system for turning: data model, architecture, and implementation. Computer Aided Design, 38(6), 677-688. https://doi.org/10.1016/j.cad.2006.02.006

[10] Xu, X. W. (2006). Realization of STEP-NC enabled machining. Robotics and Computer-Integrated Manufacturing, 22(2), 144-153. https://doi.org/10.1016/j.rcim.2005.02.009

[11] Habeeb, S. \& Xu, X. (2009). A novel CNC system for turning operations based on a high-level data model. The International Journal of Advanced Manufacturing Technology, 43(3-4), 323-336. https://doi.org/10.1007/s00170-008-1718-8

[12] Minhat, M., Vyatkin, V., Xu X., Wong S. \& Al-Bayaa, Z. (2009). A novel open CNC architecture based on STEP-NC data model and IEC 61499 function blocks. Robotics and Computer-Integrated Manufacturing, 25(3), 560-569. https://doi.org/10.1016/j.rcim.2008.03.021

[13] Lan, H. B., Liu, R. L. \& Zhang, C. R. (2007). Research on the intelligent CNC system based on STEP-NC. China Mechanical Engineering, 18(6), 692-696.

[14] Lee, W., Bang, Y. B., Ryou, M. S., Kwon, W. H. \& Jee, H. S. (2006). Development of a PC-based milling machine operated by STEP-NC in XML format. International Journal of Computer Integrated Manufacturing, 19(6), 593602. https://doi.org/10.1080/09511920600623674

[15] Delta Tau. (2016). Machine and Motion controllers. Retrieved from http://www.deltatau.com/DT Products/ MachineAndMotionProductList.aspx (2016, June 04)

[16] Gao, S. M. (1998). A survey of automatic feature recognition. Chinese Journal of Computers, 21(3), 281-288.

\section{Contact information:}

Yu ZHANG, Assoc. Prof. Ph.D.

(Corresponding author)

1) School of Mechanical Engineering and Automation, Northeastern University

NO. 3-11, Wenhua Road, Heping District, Shenyang, China, 110819

2) Shenyang Blower Works Group Corporation

No.16A, Development Road, Economic \& Technical Development Zone,

Shenyang, China, 110869

E-mail: zy4097534@126.com

\section{Qifeng ZENG, Master candidate}

School of Mechanical Engineering and Automation, Northeastern University NO. 3-11, Wenhua Road, Heping District, Shenyang, China, 110819

E-mail: 1401290952@qq.com

\section{Guodong MU, Master candidate}

School of Mechanical Engineering and Automation, Northeastern University NO. 3-11, Wenhua Road, Heping District, Shenyang, China, 110819 E-mail:2395864278@qq.com

\section{Yafei YANG, Master candidate}

School of Mechanical Engineering and Automation, Northeastern University NO. 3-11, Wenhua Road, Heping District, Shenyang, China, 110819 E-mail:1598845521@qq.com

Yutao YAN, Assoc. Prof. Ph.D.

School of Mechanical Engineering and Automation, Northeastern University NO. 3-11, Wenhua Road, Heping District, Shenyang, China, 110819 E-mail: 56115209@qq.com

Wanli SONG, Assoc. Prof. Ph.D.

School of Mechanical Engineering and Automation, Northeastern University NO. 3-11, Wenhua Road, Heping District, Shenyang, China, 110819 E-mail: 58680812@qq.com

Yadong GONG, Prof. Ph.D.

School of Mechanical Engineering and Automation, Northeastern University NO. 3-11, Wenhua Road, Heping District, Shenyang, China, 110819 E-mail: 1670390742 @qq.com 